\title{
Optical afterburner for an x-ray free electron laser as a tool for pump-probe experiments
}

\author{
E. L. Saldin, E. A. Schneidmiller, and M. V. Yurkov \\ Deutsches Elektronen-Synchrotron (DESY), Notkestrasse 85, D-22607 Hamburg, Germany
}

(Received 22 September 2009; published 5 March 2010)

\begin{abstract}
We propose a new scheme for two-color operation of an x-ray self-amplified spontaneous emission free electron laser (SASE FEL). The scheme is based on an intrinsic feature of such a device: chaotic modulations of electron beam energy and energy spread on the scale of FEL coherence length are converted into large density modulations on the same scale with the help of a dispersion section, installed behind the x-ray undulator. Powerful radiation is then generated with the help of a dedicated radiator (like an undulator that selects a narrow spectral line), or one can simply use, for instance, broadband edge radiation. A typical radiation wavelength can be as short as a FEL coherence length, and can be redshifted by increasing the dispersion section strength. In practice it means the wavelength ranges from vacuum ultraviolet to infrared. The long-wavelength radiation pulse is naturally synchronized with the x-ray pulse and can be either directly used in pump-probe experiments or cross correlated with a high-power pulse from a conventional laser system. In this way experimenters overcome jitter problems and can perform pump-probe experiments with femtosecond resolution. Additional possibilities like on-line monitoring of x-ray pulse duration (making "optical replica" of an x-ray pulse) are also discussed in the paper. The proposed scheme is very simple, cheap, and robust, and therefore can be easily realized in facilities like FLASH, European XFEL, LCLS, and SCSS.
\end{abstract}

DOI: 10.1103/PhysRevSTAB.13.030701

PACS numbers: 41.60.Cr

\section{INTRODUCTION}

Free-electron lasing at wavelengths shorter than the ultraviolet can be achieved with a single-pass, high-gain free electron laser (FEL) amplifier operating in the socalled self-amplified spontaneous emission (SASE) mode, where the amplification process starts from shot noise in the electron beam [1-3]. Present acceleration and FEL techniques allow one to generate powerful, coherent femtosecond pulses in wavelength range from vacuum ultraviolet (VUV) [4-6] through soft x-ray [7,8] to hard x-ray [9].

There is growing interest among FEL users in pumpprobe experiments with femtosecond resolution, extended into the x-ray regime [10-12]. However, synchronization of pulses from an x-ray FEL with optical pulses from a conventional laser with femtosecond accuracy is not a trivial task due to a jitter in an electron bunch arrival time. The most simple and reliable way to get around this obstacle is to produce an $\mathrm{x}$-ray pulse and a longwavelength radiation pulse by the same electron bunch $[13,14]$. If the optical pulse is sufficiently powerful, it can be directly used in a pump-probe experiment. Otherwise this long-wavelength radiation pulse can be used for a cross-correlation measurement with a powerful pulse from a conventional laser (that is used in the pumpprobe experiment) [15]. This allows one to determine a relative delay between two pulses for every shot and then to sort out experimental data using this information. Different scenarios were considered [13-17] for producing visible or infrared radiation by the same electron bunch that lases in an x-ray undulator.

Here we propose a very cheap and robust method, based on natural features of a SASE FEL. Namely, chaotic modulations of electron beam energy and energy spread on the scale of FEL coherence length are converted into large density modulations on the same scale with the help of a dispersion section, installed behind the x-ray undulator (see Fig. 1). Powerful radiation is then generated with the help of a dedicated radiator (like an undulator that selects a narrow spectral line), or one can simply use, for instance, broadband edge radiation or synchrotron radiation from a bend.

The paper is organized as follows. In Sec. II we describe the physical effect of producing long-range microbunching and introduce simple scaling relations. In Secs. III, IV, and $\mathrm{V}$ we illustrate a possible application of this effect to different facilities (European XFEL [12], FLASH [7,8], LCLS [9]) considering different solutions for dispersion sections and radiators. In Sec. VI we draw the readers' attention to an additional possibility of using the consid-

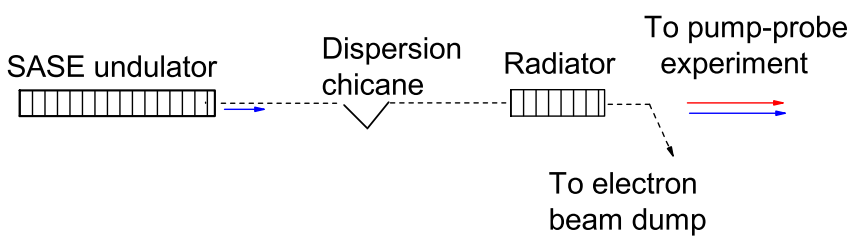

FIG. 1. (Color) Scheme of the afterburner for pump-probe experiments at an x-ray FEL. 

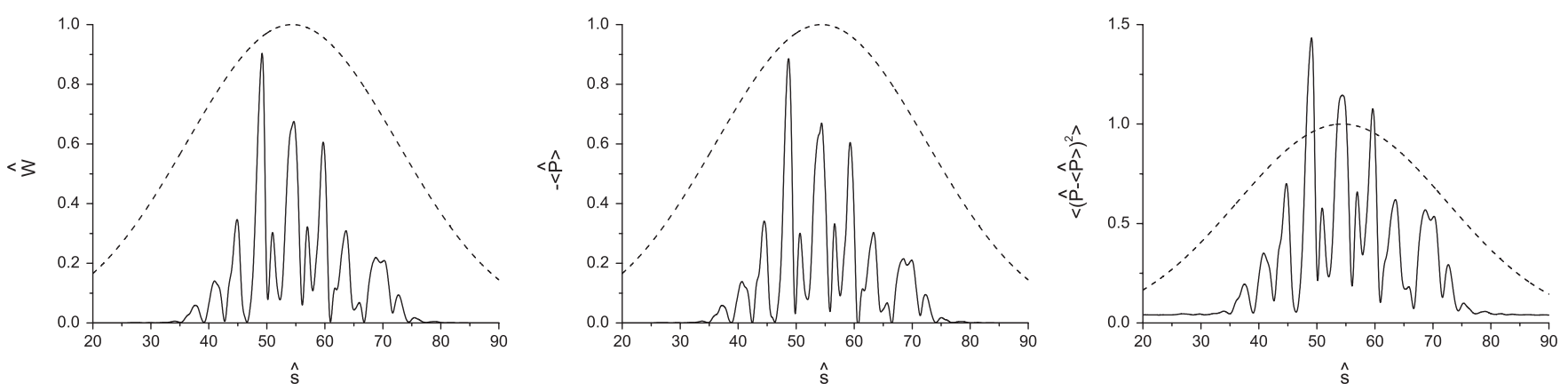

FIG. 2. Scaled radiation power (left), beam energy loss (middle), and the variance of energy distribution (right) versus scaled position along the bunch for a SASE FEL operating close to saturation (two field gain lengths before saturation). Undisturbed energy spread is $\hat{\Lambda}_{T}=0.2$. Simulations were performed with the 1D version of the code FAST [20]. The electron bunch profile is shown in dashed lines.

ered effect, namely on-line monitoring of x-ray pulse duration by translating its width and shape into optical range, i.e., making an "optical replica" of an x-ray pulse.

\section{EFFECT DESCRIPTION}

SASE FEL is a high-gain FEL amplifier starting up from shot noise in an electron beam. The properties of such a device are described in detail in [18]. On top of density and energy modulations in the electron beam on the scale of a resonant wavelength $\lambda_{r}$, there are long-scale envelope modulations. The latter modulations have a typical scale of FEL coherent length, $l_{c} \simeq \lambda_{r} /(2 \pi \rho)$, where $\rho$ is the FEL parameter [19]. There are mean energy losses in the electron beam, modulated on the same scale, $l_{c}$. However, there are no spectral components of beam density in the range $\lambda \simeq l_{c}$ (more precisely, there can be small modulations of the order of $\rho \simeq 10^{-4}-10^{-3}$ when SASE FEL reaches saturation). Here we propose a simple trick which can be used in order to get large density modulations of the order of unity.

Let us consider a continuous electron beam of which longitudinal phase space at the exit of the FEL undulator can be described by a distribution function $f(P, s)$, where $P=E-E_{0}$ is energy deviation from a nominal value, and $s$ is the coordinate along the bunch. It is convenient to use scaled variables $\hat{P}=P /\left(\rho E_{0}\right)$ and $\hat{s}=\rho k_{r} s$, where $k_{r}=$ $2 \pi / \lambda_{r}$. Before FEL interaction we assume the function $f(\hat{P}, \hat{s})$ to be independent of $\hat{s}$, i.e., to have distribution in $\hat{P}$ with the rms width $\hat{\Lambda}_{T}$ which is constant along the beam. In the following we will assume that $\hat{\Lambda}_{T} \ll 1$ since it is usually the case. The distribution function is normalized such that after integration over $\hat{P}$ one gets unity for the unmodulated beam case. The FEL process modifies the distribution function: there are energy and density modulations on the scale of $\lambda_{r}$ with a chaotically changing envelope on the scale of $l_{c}$ (i.e. when $\Delta \hat{s} \simeq 1$ ), and a mean energy loss on the same scale. Note that FELinduced energy modulation and mean energy loss are typically $\hat{P} \simeq 1$ at saturation and $\hat{P} \ll 1$ in the exponential gain regime before saturation. For illustration we present in Fig. 2 some results of numerical simulations (for a finite bunch length). FEL radiation power $W$ is scaled as follows: $\hat{W}=W /\left(\rho W_{b}\right)$, where $W_{b}$ is the electron beam power. FEL operates close to saturation (two field gain lengths before saturation). ${ }^{1}$

Let us install behind the SASE undulator a dispersive element (like a simple four-bend chicane) with longitudinal dispersion characterized by matrix element $R_{56}$. After the beam passes the dispersive section, a particle's coordinate changes such that one should use the substitution $s \rightarrow s-R_{56} P / E_{0}$ in the distribution function. In scaled variables this would read $f\left(\hat{P}, \hat{s}-\hat{P} \hat{R}_{56}\right)$, where $\hat{R}_{56}=$ $\rho^{2} k_{r} R_{56}$. Even a relatively small strength of the dispersion section is sufficient to completely smear short-scale modulations due to the energy spread. The condition for that can be formulated as $\hat{\Lambda}_{T} \hat{R}_{56} \gg \rho$, and it will always be fulfilled in the rest of the paper. Therefore, after the dispersion section we only deal with long-scale modulations. Let us first consider the case when $\hat{R}_{56} \ll 1$. In this case the distribution function can be expanded as follows:

$$
\begin{aligned}
f\left(\hat{P}, \hat{s}-\hat{P} \hat{R}_{56}\right) \simeq & f(\hat{P}, \hat{s})-\frac{\partial f}{\partial \hat{s}} \hat{P} \hat{R}_{56}+\frac{1}{2} \frac{\partial^{2} f}{\partial \hat{S}^{2}} \hat{P}^{2} \hat{R}_{56}^{2} \\
& +\cdots
\end{aligned}
$$

Note that the distribution function before the dispersion section is differentiated here, but the short-scale modulations are assumed to be smeared as discussed above. Integrating over $\hat{P}$ (and exchanging differentiation and integration) we get current modulation

\footnotetext{
${ }^{1}$ Note that in the exponential gain regime the patterns of radiation power and of beam energy loss for a given shot are identical (with a small shift $\Delta \hat{s} \ll 1$ ). This also holds, with minor distortions, for the presaturation regime, shown in Fig. 2. At saturation point and beyond the identity is lost.
} 

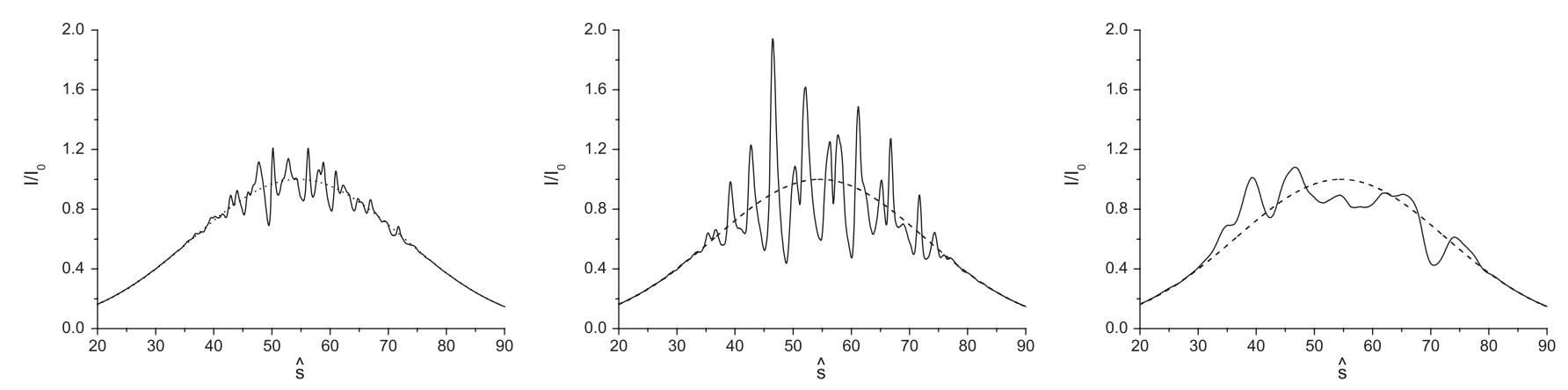

FIG. 3. Modulation of current in the electron beam, modified by FEL interaction as shown in Fig. 2, after passing the dispersion section with $\hat{R}_{56}=0.2$ (left), $\hat{R}_{56}=1$ (middle), and $\hat{R}_{56}=5$ (right). The undisturbed energy spread is $\hat{\Lambda}_{T}=0.2$. The current profile for $\hat{R}_{56}=0$ is shown in dashed lines.

$$
I / I_{0} \simeq 1-\hat{R}_{56} \frac{\partial}{\partial \hat{s}} \int \hat{P} f d \hat{P}+\frac{\hat{R}_{56}^{2}}{2} \frac{\partial^{2}}{\partial \hat{s}^{2}} \int \hat{P}^{2} f d \hat{P}+\cdots
$$

which can be rewritten as

$$
I / I_{0} \simeq 1-\hat{R}_{56} \frac{\partial\langle\hat{P}\rangle}{\partial \hat{S}}+\frac{\hat{R}_{56}^{2}}{2} \frac{\partial^{2}\left\langle\hat{P}^{2}\right\rangle}{\partial \hat{S}^{2}}+\cdots
$$

A linear term here is connected with the derivative of beam energy loss due to the SASE process. The secondorder term depends on induced rms energy spread and mean energy loss. At saturation $\langle\hat{P}\rangle$ and $\left\langle\hat{P}^{2}\right\rangle$ are typically of the order of 1 ; they change on a typical scale of coherence length $\Delta \hat{s} \simeq 1$, so that current modulation is of the order of $\hat{R}_{56}$. Although Eq. (1) is not valid when $\hat{R}_{56} \simeq 1$, one can extrapolate the tendency and estimate that in this case one can reach very strong current modulation, on the order of 1 . This is confirmed by numerical simulations performed with 1D version of the code FAST (see Fig. 3). One can also notice that for small values of $\hat{R}_{56}$ the current modulation pattern remains unchanged while its amplitude linearly increases with $\hat{R}_{56}$, as it follows from Eq. (1). When $\hat{R}_{56} \simeq 1$ the pattern is modified due to high-order terms. When $\hat{\Lambda}_{T} \hat{R}_{56} \simeq 1$, the smearing effect due to the energy spread becomes essential, and the spectrum is significantly redshifted.

Since we deal with a stochastic process, the spectrum of the density modulation as well as the spectrum of radiation, produced by such a beam in a radiator, have a spiky structure, and the pattern changes from shot to shot. Correspondingly, the radiation pulse energy fluctuates shot to shot: the more narrow bandwidth of a radiator, the stronger the fluctuations. If the SASE FEL operates in linear regime and in a long-pulse limit [18], fluctuations of pulse energy from a long-wavelength radiator would scale as $1 / \sqrt{\Delta \omega_{\text {rad }} T}$ for $\Delta \omega_{\text {rad }} \gg 1 / T$, where $\Delta \omega_{\text {rad }}$ is the radiator bandwidth and $T$ is SASE pulse duration. If the SASE FEL reaches saturation then the fluctuations are reduced.

\section{APPLICATION TO EUROPEAN XFEL}

Let us consider a possible operation of long-wavelength afterburner behind the SASE1 undulator of the European XFEL. To be specific, we assume that it operates at $0.1 \mathrm{~nm}$. Electron energy is $17.5 \mathrm{GeV}$, peak current is $5 \mathrm{kA}$, normalized emittance is $1.4 \mathrm{~mm} \mathrm{mrad}$, and local energy spread is 1.5 MeV. Other parameters of the electron beam as well as parameters of the undulator and of FEL radiation can be found in [12]. The simulations of the FEL process were performed with three-dimensional, time-dependent code FAST [20]. Then particles' positions in the bunch were changed in accordance with their energies and applied $R_{56}$. Parameter $\rho$ in the considered case is equal to $4 \times$ $10^{-4}$. Thus, it is easy to calculate that the value of $R_{56}=$ $100 \mu \mathrm{m}$ corresponds to the scaled parameter $\hat{R}_{56}=1$. As a technical realization of the dispersion section for XFEL we consider a simple four-bend chicane with a bend length of $1 \mathrm{~m}$ and a total length of $10 \mathrm{~m}$. In Figs. 4 and 5 (left plot), one can see chaotic modulations of the beam current for the case when $R_{56}$ of the chicane is equal to $50 \mu \mathrm{m}$. For smaller values of the $R_{56}$, the pattern remains the same but the amplitude reduces proportionally. For larger values of the $R_{56}$, the pattern gets strongly modified as one can see from Fig. 5, the modulations have longer scale. Figures 4 and 5 are calculated for a given shot. It is worth mentioning

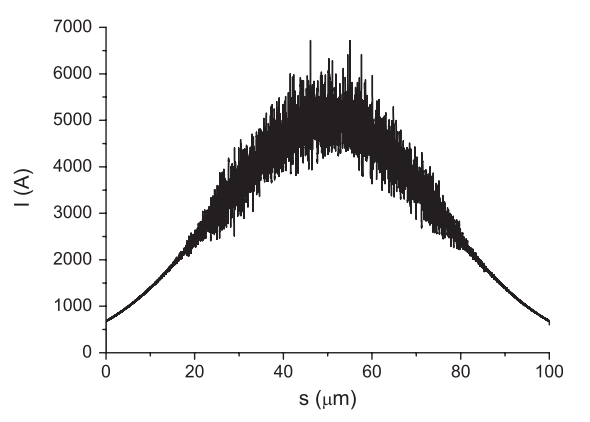

FIG. 4. Modulation of current in the beam passed SASE1 undulator [12] and a dispersion section with $R_{56}$ equal to $50 \mu \mathrm{m}$. 

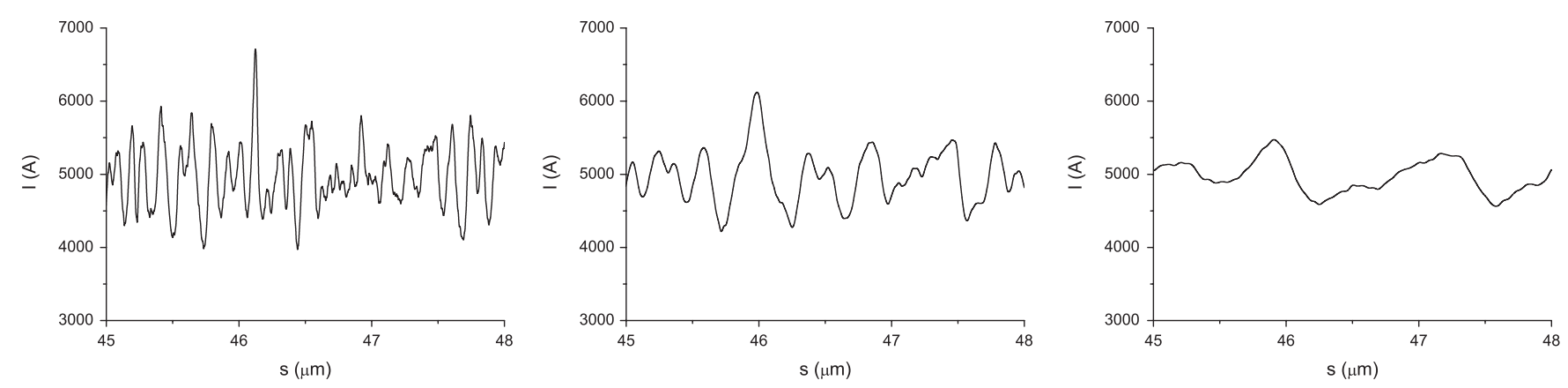

FIG. 5. Modulation of current in the beam passed SASE1 undulator and a dispersion section with $R_{56}$ equal to $50 \mu \mathrm{m}$ (left plot, enlarged fraction of Fig. 4), $200 \mu \mathrm{m}$ (middle plot), and $500 \mu \mathrm{m}$ (right plot).

that the pattern would change shot to shot since SASE is a stochastic process.

Note that the operation of SASE1 and consequent conversion to long-scale density modulations were calculated for a rather conservative value of normalized emittance $1.4 \mathrm{~mm}$ mrad [12] of electron beam. In that case only core in transverse distribution of electron bunch efficiently contributes to lasing, so that a relatively large part of the beam is unmodulated and does not contribute to long-scale density modulations after conversion. That is why the amplitude of modulations is limited to $10 \%-20 \%$ level in our calculations. In recent experiments with the first hard x-ray FEL [9], however, a routine operation with a significantly smaller emittance, $0.4 \mathrm{~mm}$ mrad, was demonstrated. In such a case a larger part of the electron beam is involved in SASE process, so that larger long-scale modulations behind a dispersive section can be expected.

In Fig. 6 we present an ensemble average modulus of the bunch form factor $|F(\lambda)|$ (Fourier transform of the bunch profile). This is a useful notion because energy spectral density of radiation produced by a bunch in any radiator is given by

$$
p(\lambda)=p_{1}(\lambda)\left[N_{e}+N_{e}\left(N_{e}-1\right)|F(\lambda)|^{2}\right]
$$

where $p_{1}(\lambda)$ is the energy spectral density produced by a single electron, and $N_{e}$ is the number of electrons in the bunch. The linear in $N_{e}$ term gives usual incoherent radiation (spontaneous emission). Thus, a modulated bunch radiates by a factor $N_{e}|F(\lambda)|^{2}$ more energy at a given wavelength than an unmodulated bunch. Here we consider the bunch with the charge of $1 \mathrm{nC}\left(N_{e}=6.2 \times 10^{9}\right)$ so that one can expect an enhancement over spontaneous emission by a factor up to $4 \times 10^{4}$, using $|F(\lambda)|$ from Fig. 6 .

One should not be surprised by the fact that the amplitude of modulations in Figs. 4 and 5 is typically 10\%-20\%, while the form factor is much smaller. For a monochromatic modulation the value of form factor would indeed have been of the order of 0.1. Chaotic spikes, however, add randomly to the Fourier transform and the result is suppressed roughly by a square root of the number of spikes, and the spectrum is broadband. One should also note here that single shot form factors are also chaotically modulated, while the smooth curves in Fig. 6 are the result of statistical averaging.

Form factors for different values of $R_{56}$ are presented in Fig. 6. For $R_{56}=50 \mu \mathrm{m}$ the high-frequency cutoff is at the wavelength about $100 \mathrm{~nm}$ which coincides with the coherence length of SASE FEL operating at $0.1 \mathrm{~nm}$ (note that one can have a significant enhancement over spontaneous emission even at $40-50 \mathrm{~nm}$ ). For larger values of the $R_{56}$, one can observe a redshift. Thus, by varying $R_{56}$ one can easily optimize the form factor in the wavelength range from VUV to infrared.
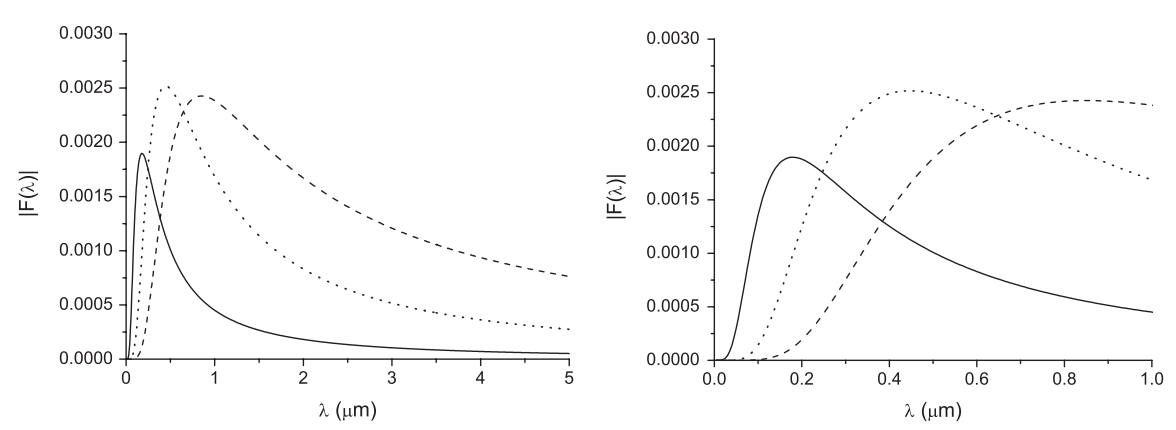

FIG. 6. Ensemble average modulus of the bunch form factor versus wavelength for SASE1 afterburner, and an enlarged fraction of this plot, for the $R_{56}$ of the dispersion section equal to $50 \mu \mathrm{m}$ (solid), $200 \mu \mathrm{m}$ (dotted), and $500 \mu \mathrm{m}$ (dashed). 
Let us now discuss how to produce radiation that can be transported to the experimental hall and used in pumpprobe experiments as described in [15]. In principle, one can make use of edge radiation [15]. In that case a broadband radiation would be produced (which can be then filtered if necessary). The estimated number of photons per shot would be on the order of $10^{12}$. The spectrum width would correspond approximately to the width of a form factor curve in Fig. 6. The distance between the end of the SASE1 undulator and a bending system downstream is about $200 \mathrm{~m}$ which is comparable to a formation length $\lambda \gamma^{2}\left(\gamma \simeq 3 \times 10^{4}\right.$ being relativistic factor, $\left.\lambda=\lambda / 2 \pi\right)$ for a visible part of the spectrum. For longer wavelengths, a few $\mu \mathrm{m}$, divergence of the radiation would increase, and the spectral properties of radiation at the experiment would depend on a design of the optical transport system.

We also consider here an alternative radiator, namely a long-period undulator (similar to that installed in FLASH $[13,21])$. Let us consider as an example, a ten-period undulator with a period length $70 \mathrm{~cm}$ and a maximum field $1.2 \mathrm{~T}$. For the electron energy of $17.5 \mathrm{GeV}$, one can tune the resonant wavelength between $50 \mathrm{~nm}$ and $1 \mu \mathrm{m}$ by changing the undulator field. To calculate radiation pulse energy, one should use the fact that in a planar undulator with large $\mathrm{K}$-value 0.011 photons per electron are radiated spontaneously within a central cone (given by $\sqrt{\lambda / L_{w}}$, where $L_{w}$ is the undulator length). Then, using Eq. (2) and Fig. 6, we end up with Fig. 7, from which we see that pulse energies are in the $\mu \mathrm{J}$ range within a central cone and with spectral bandwidth $10 \%$. Estimated shot-to-shot fluctuations will be below $10 \%$.

If we compare the two scenarios of producing radiation, the latter promises relatively narrow bandwidth (with wavelength tunability) with roughly the same number of photons as in the former case within a wide band. In other words, spectral brightness of the undulator radiation is larger by 1 order of magnitude in the considered case. Note that transverse coherence is guaranteed for both sources (since the condition $\epsilon \ll \lambda, \epsilon$ being electron beam emittance, is well satisfied). However, source size

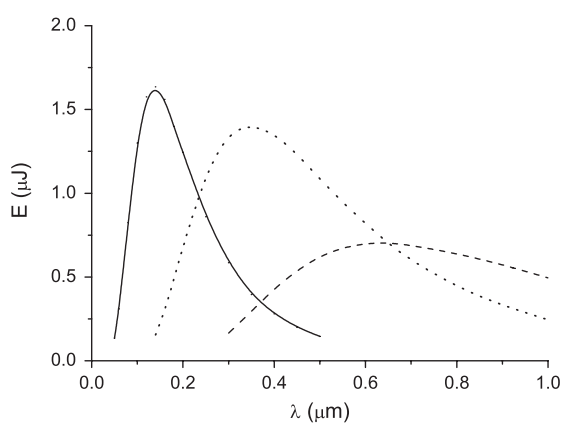

FIG. 7. Radiation pulse energy within a central cone of the SASE1 afterburner undulator versus wavelength for the $R_{56}$ of the dispersion section equal to $50 \mu \mathrm{m}$ (solid), $200 \mu \mathrm{m}$ (dotted), and $500 \mu \mathrm{m}$ (dashed). and divergence are very much different in the two cases. In the case of visible edge radiation the divergence is about $1 / \gamma$. In fact, exact calculation in [15] gives a half angle of $50 \mu \mathrm{rad}$ for the first maximum of edge radiation. In the case of undulator radiation the central cone $\sqrt{\lambda / L_{w}}$ is in the range 100-400 $\mu \mathrm{rad}$ for the wavelength range shown in Fig. 7.

\section{APPLICATION TO FLASH}

FLASH is the SASE FEL operated as the user facility in the wavelength range $6.5-50 \mathrm{~nm}[5,7,8]$. There is a nineperiod FIR (far infrared) undulator $[13,21]$ installed downstream of the main SASE undulator. Its wavelength range spans from sub- $\mu \mathrm{m}$ up to $200 \mu \mathrm{m}$. The $R_{56}$ of an undulator is given by a simple formula: $R_{56}=2 N_{w} \lambda_{r}$, where $N_{w}$ is the number of periods and $\lambda_{r}$ is the resonant wavelength. Thus, the $R_{56}$ of this undulator can be tuned from 0 to a few $\mathrm{mm}$, and it can serve in the considered scheme as a dispersive section and a radiator (radiating at higher harmonics) at the same time. In addition, edge radiation can be produced downstream of this undulator (there is a few meter drift space between the undulator and the beam dump, so that there is enough space for formation of such radiation in the visible and the near-infrared range). We illustrate with a numerical example a possible operation of an afterburner. We consider lasing at $7 \mathrm{~nm}$ and use in simulations an electron beam with the parameters close to those used in [7] but rescaled to $1 \mathrm{GeV}$. The bunch with the charge of $0.5 \mathrm{nC}$ consists of a few ps long low-current tail and a short high-current leading peak (shown in Fig. 8 in dots) that produces FEL radiation. We illustrate conversion of FEL-induced energy modulations to modulations of beam current by applying the $R_{56}=250 \mu \mathrm{m}$ (which corresponds to tuning of the FIR undulator to $14 \mu \mathrm{m}$ resonant wavelength). In Fig. 8, one can see a realization of such modulations in the time domain, and Fig. 9 shows form factor, corresponding to this specific shot. Note that the form factor of undisturbed bunch decays at 5-10 $\mu \mathrm{m}$, and

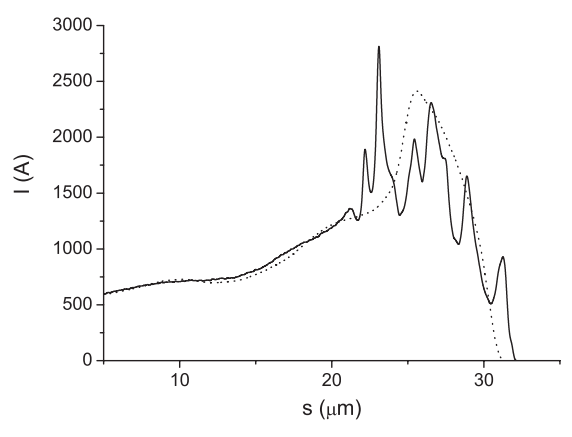

FIG. 8. Modulation of current in the head of electron bunch lasing at $7 \mathrm{~nm}$ in the VUV undulator of FLASH and passing FIR undulator with $R_{56}$ equal to $250 \mu \mathrm{m}$ (solid), and with $R_{56}=0$ (dots). Only the small part of the bunch is shown; the bunch head is on the right. 

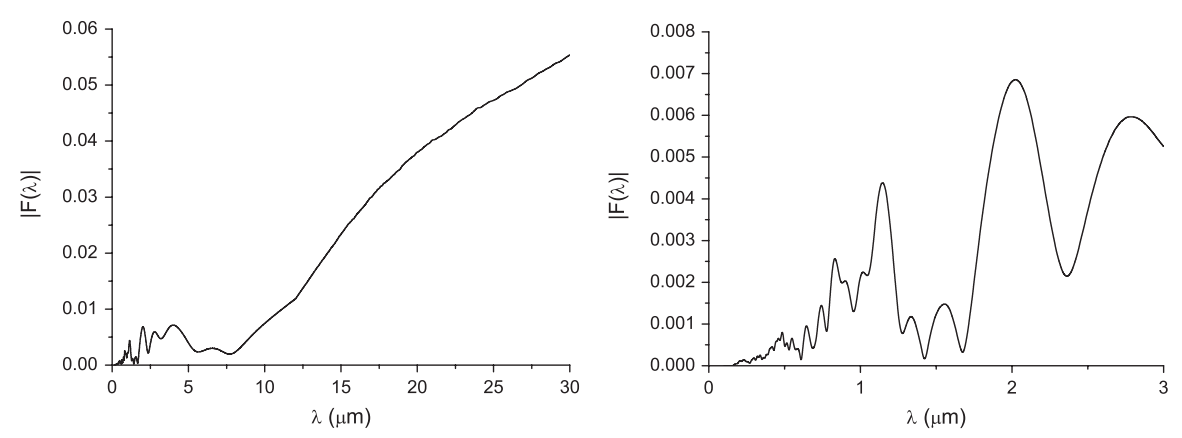

FIG. 9. Modulus of the bunch form factor (corresponding to realization in the time domain shown in Fig. 8) versus wavelength, and an enlarged fraction of this plot.

the effect, described in this paper, allows one to extend the possibility to produce powerful radiation to $1 \mu \mathrm{m}$ and below. ${ }^{2}$ For instance, in a given example the radiation in the range $0.7-1 \mu \mathrm{m}$ would be about 4 orders of magnitude above the spontaneous emission level. Since it is synchronized with soft $\mathrm{x}$-ray radiation, it can be used for timing purposes in a pump-probe experiment on a $10 \mathrm{fs}$ time scale.

\section{DUMP DIPOLE AS A BUNCHER AND A RADIATOR}

After passing the x-ray undulator the electron beam is directed towards beam dump. A bending system upstream of the dump, consisting of one or several dipoles, has dispersive properties, on the one hand, and forces the beam to radiate, on the other hand. We note that under some circumstances the beam can be efficiently bunched on the scale of the FEL coherence length and then produce coherent synchrotron radiation (CSR). In this case the scheme in Fig. 1 becomes trivial (no dispersive chicane and no dedicated radiator). Although such a configuration is not very efficient, it is the most cost-saving solution.

Longitudinal dispersion in a dipole increases as follows: $R_{56}(z)=R \theta^{3}(z) / 6$, where $z$ is the longitudinal coordinate along the beam line, and $\theta(z)=z / R$ is a current bending angle, $R$ is the bending radius. It means that density modulation also increases as a third power of $z$ (assuming here $\hat{R}_{56} \ll 1$ ). This happens until it gets suppressed by a transverse beam size $\sigma_{\perp}$ in the bending plane due to the $R_{51}$ effect [22-24], i.e., when $\theta(z) \sigma_{\perp} \simeq \lambda$. Thus, maximum density bunching appears at the position $z_{m} \simeq$ $R \lambda / \sigma_{\perp}$, and the corresponding longitudinal dispersion is $R_{56} \simeq R \lambda^{3} /\left(6 \sigma_{\perp}^{3}\right)$. It is interesting to note a sharp dependence of radiated power on the transverse beam size and, therefore, on emittance $\epsilon$ and beta function $\beta$ in a bending plane. Indeed, maximum density bunching scales as $z_{m}^{3}$, and the maximum radiation power as $z_{m}^{6} \propto \sigma_{\perp}^{-6}=(\epsilon \beta)^{-3}$. In this consideration we assumed that $\left(\lambda R^{2}\right)^{1 / 3} \ll z_{m}<$ $L_{d}$, where $L_{d}$ is the dipole length and $\left(\lambda R^{2}\right)^{1 / 3}$ is the

\footnotetext{
${ }^{2}$ Note that some observations of such a kind were done at FLASH, and now we can hope for systematic studies.
}

formation length of coherent synchrotron radiation. The strongest intensity burst would sweep around the angle $\lambda / \sigma_{\perp}$ in the bending plane with the divergence $(\lambda / R)^{1 / 3}$ in the other plane. Since one deals with broadband density modulation, shorter wavelengths have maxima at smaller bending angles, i.e., one has a rainbowlike phenomena.

As an example, let us consider the bending system of the Linac Coherent Light Source [9,10]. It consists of several dipoles; the first one is $1.5 \mathrm{~m}$ long, and its bending angle is about $40 \mathrm{mrad}$. Let us assume that the SASE FEL saturates at $0.15 \mathrm{~nm}$ with electron energy $13.6 \mathrm{GeV}$ and normalized slice emittance $0.4 \mathrm{~mm}$ mrad [9]. Let us consider CSR at the wavelength $\lambda=700 \mathrm{~nm}$, assuming beta function $\beta=$ $10 \mathrm{~m}$ in the bending plane. In that case we estimate that $z_{m} \simeq 35 \mathrm{~cm}$ (while the radiation formation length is about $5 \mathrm{~cm}$ ), the corresponding bending angle is $10 \mathrm{mrad}$, and longitudinal dispersion is $R_{56} \simeq 6 \mu \mathrm{m}$. One can expect a burst of CSR with an opening angle $(\lambda / R)^{1 / 3} \simeq 1.5 \mathrm{mrad}$ and a significant pulse energy (a few orders of magnitude higher than that of incoherent synchrotron radiation at a given wavelength).

\section{ON-LINE MONITORING OF X-RAY PULSE DURATION}

Measurement of duration of an x-ray pulse from a SASE FEL is a difficult task. One can obtain indirect information from spectral measurements, fluctuation analysis [4,5,7], etc. Recently, at FLASH there were attempts of measuring width and shape of extreme ultraviolet (XUV) femtosecond pulses in time domain $[25,26]$. Using the effect considered in this paper, one can make a considerable step in this direction. Indeed, this effect leads to "visualization" of x-ray pulse, i.e., translating its width and shape into optical range, where direct on-line measurement of femtosecond pulses is possible with the help of frequencyresolved optical gating (FROG) [27] or similar techniques. FROG works very well in the IR, visible, and UV, and can measure temporal and spectral distributions of extremely complex pulses. FROG was proposed to be used in an optical replica synthesizer (ORS) [28], a device for diagnostics of femtosecond electron bunches. Recently, the 

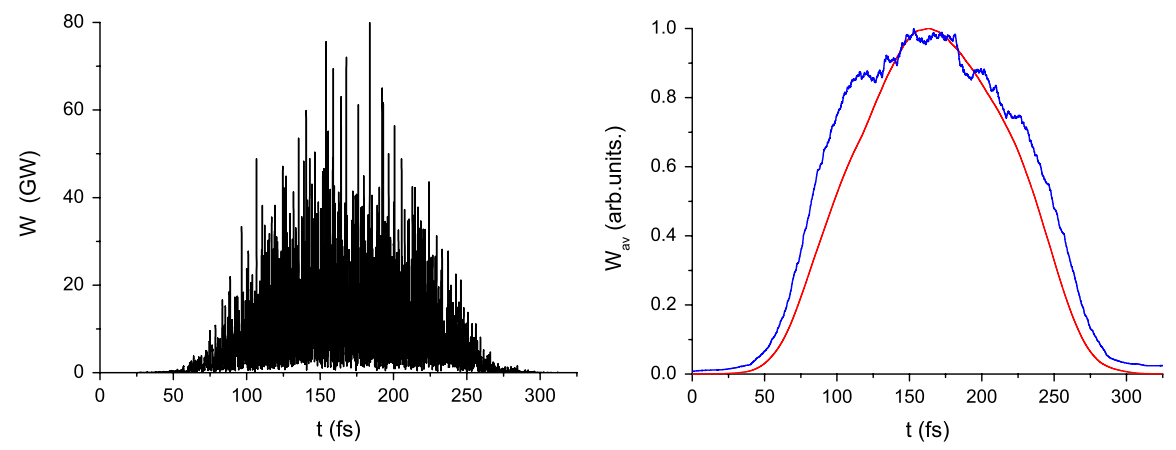

FIG. 10. (Color) Left: Power distribution in a single x-ray pulse from the SASE1 undulator [12]. This shot was used to calculate beam modulations in Fig. 4. Right: Averaged power distribution (red) and its reconstruction (blue), see the text for details.

ORS setup was successfully tested [29] at FLASH. In this paper we discuss a possibility of making an optical replica of an x-ray pulse.

Let us consider a long-pulse case (much longer than FEL coherence length). The simplest measurement, aiming at ensemble averaged shape of the SASE FEL radiation pulse, can be described as follows. When the SASE FEL operates in the exponential gain regime and just before the saturation, the average shape of the radiation pulse is imprinted in the electron bunch, i.e., it is repeated by the shape of beam energy loss. After a dispersion section with a small $\hat{R}_{56}$ this shape is transferred into the density modulation. If the bandwidth of a radiator is much larger than the inverse duration of the FEL pulse, $\Delta \omega_{\text {rad }} \gg 1 / T$, the ensemble averaged shape of field amplitude of the optical radiation pulse would repeat the averaged shape of the $\mathrm{x}$ ray intensity pulse. The shape of the field amplitude of an optical pulse can be measured by FROG. ${ }^{3}$ Then one can average the traces over the ensemble of realizations. Alternatively, one can do adjacent averaging of a single pulse (moving a small time window along the trace) at the expense of some remaining modulations.

It is interesting to see how this method would work in a somewhat more complicated (but practically important) situation: FEL operates at saturation, and $\hat{R}_{56}$ is not too small, otherwise optical intensity might be too weak. As an example let us consider again the case of the SASE1 undulator of the European XFEL. In Fig. 10 (left) we show a single FEL pulse-this specific realization was used to calculate Fig. 4; there we applied $\hat{R}_{56}=0.5$. Then the optical radiation pulse is produced by a bunch, presented in Fig. 4. For the following we have to assume a bandwidth of a radiator, optical transport system, etc. To be specific, we let the bunch from Fig. 4 go through a bandpass filter between 250 and $350 \mathrm{~nm}$ to simulate amplitude of the electric field of the optical pulse. Finally, we used adjacent averaging with a 20 fs time window. The result is shown in Fig. 10 in blue, and is compared with the original

${ }^{3}$ FROG shows intensity trace in time domain, so that one should take a square root of it averaged FEL intensity (shown in red). Some 10\%-20\% broadening can be explained by FEL saturation effects, a significant $\hat{R}_{56}=0.5$, and adjacent averaging procedure. Nevertheless, such an accuracy of pulse width determination would be a great step towards detailed diagnostics of $\mathrm{x}$-ray pulses from SASE FELs.

Finally, let us point out a potential possibility of measuring fine structure of a specific pulse from an x-ray FEL. Indeed, as one can see from Fig. 2, a pattern of energy modulation is a copy of an intensity pattern in FEL pulse, as soon as the FEL operates before saturation. For small $\hat{R}_{56}$ the derivative of this pattern gives the density modulation. Then this beam radiates, and the information about $\mathrm{x}$-ray pulse structure is transferred into the optical range, where it can be extracted much easier than directly in the $\mathrm{x}$-ray range. Unfortunately, at saturation the identity between FEL intensity and energy modulation is lost, so that one can only measure ensemble averaged intensity as described above. As for the possibility to measure relatively short SASE pulses (a few intensity spikes), we estimate that the accuracy of the method would be reduced but it would still be useful if there is a lack of alternatives.

\section{DISCUSSION}

In this paper we have described a novel effect associated with an x-ray FEL operation. There can be different applications of this effect (as well as some drawbacks [30]), and we have chosen two of them to illustrate an importance of the optical afterburner. In our opinion, a pump-probe (PP) experiment could be the most interesting application of the proposed scheme. Indeed, the time jitter between x-ray pulse and a pulse from an optical laser is the main obstacle on the way to PP experiments with femtosecond resolution. The jitter originates in the electron beam formation system and amounts, for instance, to 0.5 ps [31] at FLASH, while durations of soft $\mathrm{x}$-ray pulses are about $10 \mathrm{fs}[7,8]$. The most reliable way to get around this obstacle is to produce an $\mathrm{x}$-ray pulse and a long-wavelength radiation pulse by the same electron bunch $[13,14]$. In a recent experiment at FLASH [26], a soft x-ray pulse and a coherent far infrared 
radiation pulse, produced in a dedicated undulator by the same electron bunch [13], were transported by about $100 \mathrm{~m}$ down separate beam lines $[8,21]$ and combined in an experimental chamber. Measured time jitter between two pulses was $5 \mathrm{fs}$ (rms) [26]. This experiment has confirmed validity and importance of the concept "single electron bunch-two colors for PP experiments".

In this paper we have proposed a new scheme that allows one to produce a synchronized x-ray pulse and an optical pulse without external manipulations with electron beam (no lasers, etc.). The scheme relies on natural features of SASE FELs and is very simple, cheap, and robust. Although in this paper we have mainly considered design parameters of the European XFEL with relatively long hard X-ray pulses (100 fs), the scheme will also work with much shorter, single-digit femtosecond pulses. It is worth mentioning that a dispersive element behind the $\mathrm{x}$ ray undulator does not spoil synchronization between two pulses. Indeed, for a typical $R_{56} \simeq 100 \mu \mathrm{m}$ and an expected energy jitter on the order of $10^{-4}$, an induced time jitter would be about 30 attoseconds only.

We have not considered in this paper many details connected with PP experiments themselves. Of course, both pulses must be transported to an experiment, where experimenters should be able to delay one of the pulses depending on the type of the experiment (optical pump-$\mathrm{x}$-ray probe or vice versa). Note that $\mathrm{x}$-ray optics allows for such delays without high losses of intensity. An optical pulse can be used directly, if its intensity is sufficient for a given experiment, otherwise it can be first amplified in a conventional laser amplifier. Alternatively, one can use a dedicated high-power laser to generate optical pulses for PP experiments. These pulses can be cross correlated with the pulses from the afterburner, so that a relative delay is measured for every shot, and then the experimental data is sorted out with the help of this information. It was not the goal of this paper to discuss all the aspects of possible pump-probe experiments, we have only demonstrated a possibility of jitter-free generation of x-ray and optical pulses.

In this paper we have assumed that the electron bunch before FEL interaction is smooth and sufficiently long, i.e., that it does not contain significant spectral components in optical range. However, in real accelerators, producing high-brightness electron beams for x-ray FELs, a microbunching instability [32,33] can take place. The most dangerous effect is the longitudinal space charge driven microbunching instability $[33,34]$. The experimental observations of such an instability $[35,36]$ indicate that amplification of microbunching takes place in infrared and visible ranges, and manifests itself, in particular, in a strong coherent optical transition radiation (COTR). The question arises whether or not the microbunching instability can interfere with the operation of the optical afterburner, proposed in this paper. At this point we have to mention that a laser heater was suggested [34] to suppress microbunching instability since the latter is sensitive to a local energy spread. A particularly clever design of a laser heater [37] was then developed for the Linac Coherent Light Source (LCLS). Recently, it was built and experimentally tested [38] during the commissioning and successful operation of LCLS. It was found that the heater significantly improves FEL performance. Concerning COTR, "the laser heater suppresses these coherent signals by orders of magnitude in many cases but does not appear to completely remove a very small level of COTR after compression" [38]. This means that when the heater is applied, the remaining modulations in optical range are much smaller than the ones induced in the proposed optical afterburner (that are at the level of tens of percent) and should not confuse its applications. However, for any specific realization of this scheme this has to be checked experimentally: when the SASE process is suppressed (for instance, by missteering the beam in the undulator), the optical radiation from the afterburner must be strongly reduced. In this way, one can make sure that microbunching instability does not affect the operation of the afterburner.

[1] A. M. Kondratenko and E. L. Saldin, Part. Accel. 10, 207 (1980).

[2] Ya. S. Derbenev, A. M. Kondratenko, and E. L. Saldin, Nucl. Instrum. Methods 193, 415 (1982).

[3] J. B. Murphy and C. Pellegrini, Nucl. Instrum. Methods Phys. Res., Sect. A 237, 159 (1985).

[4] V. Ayvazyan et al., Phys. Rev. Lett. 88, 104802 (2002).

[5] V. Ayvazyan et al., Eur. Phys. J. D 37, 297 (2006).

[6] T. Shintake et al., Nat. Photon. 2, 555 (2008).

[7] W. Ackermann et al., Nat. Photon. 1, 336 (2007).

[8] K. Tiedtke et al., New J. Phys. 11, 023029 (2009).

[9] P. Emma, in Particle Accelerator Conference, Vancouver, 2009.

[10] J. Arthur et al., SLAC Conceptual Design Report No. SLAC- R593, Stanford, 2002 (see also http://wwwssrl.slac.stanford.edu/lcls/cdr).

[11] SCSS X-FEL: Conceptual design report, RIKEN, Japan, 2005 (see also http://www-xfel.spring8.or.jp).

[12] M. Altarelli et al., DESY Technical Design Report No. DESY 2006-097, Hamburg, 2006 (see also http:// xfel.desy.de).

[13] B. Faatz et al., Nucl. Instrum. Methods Phys. Res., Sect. A 475, 363 (2001).

[14] J. Feldhaus et al., Nucl. Instrum. Methods Phys. Res., Sect. A 528, 453 (2004).

[15] G. A. Geloni et al., Opt. Commun. 281, 3762 (2008).

[16] A. Zholents and W. M. Fawley, Phys. Rev. Lett. 92, 224801 (2004).

[17] E. L. Saldin, E. A. Schneidmiller, and M. V. Yurkov, in Proceedings of the 29th FEL Conference, Novosibirsk, Russia, 2007, p. 248.

[18] E. L. Saldin, E. A. Schneidmiller, and M. V. Yurkov, The Physics of Free Electron Lasers (Springer, Berlin, 2000). 
[19] R. Bonifacio, C. Pellegrini, and L. M. Narducci, Opt. Commun. 50, 373 (1984).

[20] E. L. Saldin, E. A. Schneidmiller, and M. V. Yurkov, Nucl. Instrum. Methods Phys. Res., Sect. A 429, 233 (1999).

[21] Gensch et al., Infrared Phys. Technol. 51, 423 (2008).

[22] E. L. Saldin, E. A. Schneidmiller, and M. V. Yurkov, Nucl. Instrum. Methods Phys. Res., Sect. A 490, 1 (2002).

[23] S. Heifets, G. Stupakov, and S. Krinsky, Phys. Rev. ST Accel. Beams 5, 064401 (2002).

[24] Z. Huang and K.-J. Kim, Phys. Rev. ST Accel. Beams 5, 074401 (2002).

[25] R. Mitzner et al., Phys. Rev. A 80, 025402 (2009).

[26] U. Fruehling et al., Nat. Photon. 3, 523 (2009).

[27] R. Trebino, Frequency-Resolved Optical Gating: The Measurement of Ultrashort Laser Pulses (Kluwer Academic Publishers, Boston, 2002).

[28] E. L. Saldin, E. A. Schneidmiller, and M. V. Yurkov, Nucl. Instrum. Methods Phys. Res., Sect. A 539, 499 (2005).
[29] P. Salen et al., in Proceedings of the 31st FEL Conference, Liverpool, 2009, p. 739.

[30] E. L. Saldin, E. A. Schneidmiller, and M. V. Yurkov, Proceedings of the 31st FEL Conference, Liverpool, 2009 (Ref. [29]).

[31] P. Radcliffe et al., Appl. Phys. Lett. 90, 131108 (2007).

[32] M. Borland et al., Nucl. Instrum. Methods Phys. Res., Sect. A 483, 268 (2002).

[33] E. L. Saldin, E. A. Schneidmiller, and M. V. Yurkov, Nucl. Instrum. Methods Phys. Res., Sect. A 483, 516 (2002).

[34] E. L. Saldin, E. A. Schneidmiller, and M. V. Yurkov, Nucl. Instrum. Methods Phys. Res., Sect. A 528, 355 (2004).

[35] R. Akre et al., Phys. Rev. ST Accel. Beams 11, 030703 (2008).

[36] B. Schmidt et al., in Proceedings of the 30th FEL Conference, Gyeongju, Korea, 2008, p. 397.

[37] Z. Huang et al., Phys. Rev. ST Accel. Beams 7, 074401 (2004).

[38] Z. Huang et al., Phys. Rev. ST Accel. Beams 13, 020703 (2010). 